\title{
Time Is Motor Neuron: Therapeutic Window and Its Correlation with Pathogenetic Mechanisms in Spinal Muscular Atrophy
}

\author{
Alessandra Govoni ${ }^{1}$ \& Delia Gagliardi ${ }^{1} \&$ Giacomo P. Comi ${ }^{1} \&$ Stefania Corti $^{1}$
}

\begin{abstract}
Spinal muscular atrophy (SMA) is an autosomal recessive neuromuscular disorder characterized by the degeneration of lower motor neurons (MNs) in the spinal cord and brain stem, which results in relentless muscle weakness and wasting, leading to premature death due to respiratory complications. The identification of the specific mutations in the survival motor neuron 1 (SMN1) gene that causes SMA has led to the development of experimental therapeutic strategies to increase SMN protein expression, including antisense oligonucleotides, small molecules, and gene therapy, which have so far shown promising results. The timing of therapeutic intervention is crucial since most of the degeneration in MNs occurs in the first months of life in patients with SMA type 1, which is the most severe and common form of SMA. Nevertheless, a precise temporal window for therapeutic intervention has not yet been identified. Evidence from in vivo studies in mice and large animals suggested that early therapeutic intervention for SMA correlated with better motor performance, longer survival, and, occasionally, rescue of the pathological phenotype. Indeed, the need to compensate for the loss of SMN protein function seemed to diminish during adulthood (even though repair ability after nerve injury remained impaired), suggesting the possibility of tapering the therapy administration late in the disease course. Moreover, recent clinical trials on children afflicted with SMA type 1 have shown a more rapid achievement of motor milestones and diminished disease severity when therapy was administered at an early age and earlier in the disease course. Finally, these results highlight the importance of newborn screening for SMA to facilitate early diagnosis and present the patient with available treatments while they are still in the presymptomatic stage.
\end{abstract}

Keywords Spinal muscular atrophy $\cdot$ SMA · Therapeutic window · Antisense oligonucleotides · Gene therapy

\section{Introduction}

Spinal muscular atrophy (SMA) is a hereditary and progressive neuromuscular disease that is characterized by proximal-distal muscular weakness and atrophy, which results from the degeneration of lower motor neurons (MNs) in the ventral horns of the spinal cord and in lower brainstem nuclei [1, 2]. SMA is the most common autosomal recessive disease after cystic fibrosis and is the leading genetic cause of death in infancy [3-5]. The disease incidence is about 1 in 10,000 live births, whereas the carrier status incidence is 1 in 40 to 60 depending on the ethnicity [6-8].

\footnotetext{
* Stefania Corti

stefania.corti@unimi.it

${ }^{1}$ Dino Ferrari Centre, Neuroscience Section, Department of Pathophysiology and Transplantation (DEPT), Neurology Unit, IRCCS Foundation Ca' Granda Ospedale Maggiore Policlinico, University of Milan, Via Francesco Sforza 35, 20122 Milan, Italy
}

In $98 \%$ of cases, the disease is caused by a homozygous mutation, deletion, or rearrangement in the survival motor neuron 1 (SMN1) gene on chromosome 5 (SMA5q, OMIM \#253300), which encodes the SMN protein. Only the human genome, and no other species, encodes a highly homologous copy of SMN1 called SMN2; SMN2 differs from SMN1 by a few nucleotides, of which the most critical is a $\mathrm{C}$ to $\mathrm{T}$ transition in exon 7 that causes the skipping of this exon in > 90\% of SMN2 transcripts [6]. For this reason, SMN2 mainly produces a truncated protein $(\Delta 7)$, without exon 7 , much of which is unstable and rapidly degraded [9]; moreover, SMN2 expression constitutes only $10 \%$ of the full-length fully functional SMN protein [10] and thus partially compensates for the loss of SMN1.

In SMA patients, SMN2 is the major modulator of the disease phenotype, and the number of SMN2 copies inversely correlates with the severity of the phenotype. SMA types are classified by the higher motor milestones achieved such that untreated SMA type 1 patients never acquire the ability to sit and stand, and SMA type 2 patients are able to sit but cannot 
walk; however, SMA type 3 manifests after ambulation has been acquired. Because SMN protein is essential for viability, all SMA patients have at least one copy of SMN2. The SMN protein seems to play an important role in spliceosomal snRNP biogenesis and in mRNA splicing, but the precise mechanisms by which defects in this ubiquitously expressed protein selectively affect motor neuron viability remain largely unclear [11].

In the last 20 years, there have been increasing efforts to develop a therapeutic strategy for SMA. In particular, these efforts focused on increasing the SMN protein level by delivering a wild-type copy of SMN1 to patients through either gene therapy or by modulating the transcription of the SMN2 gene using oligonucleotides or small molecules.

SMA is considered an optimal candidate disease for gene therapy for several reasons. First, without treatment, SMA would be a fatal disease. Second, the specific genetic target (SMN1) and cell population (lower MNs) affected in SMA are known. Third, toxicity from gene therapy would be minimal because SMN overexpression in tissues other than lower MNs is well tolerated and the presence of SMN2 reduces the risk of an autoimmune response. Fourth, preclinical studies can be developed owing to robust animal models for SMA and SMN cDNA length that are appropriate for gene therapy vectors. Finally, since there is an inverse correlation between the number of SMN2 copies and the severity of the disorder, strategies aimed at increasing SMN protein levels by modulating SMN2 splicing can be used. The results obtained from this study led to clinical trials that are currently ongoing and rapidly approaching the approval of the first therapy for SMA based on the use of antisense oligonucleotides (Nusinersen, Spinraza).

The SMN protein has been studied in different animal models to define its specific role and the specific timeframe that it intervenes in $\mathrm{MN}$ development. The wide variability of the SMA phenotypes, the age of onset, which in $60 \%$ of cases occurs in the first few months of life, and the fact that the vast majority of treatments seem to prevent/stop disease rather than reverse MN death emphasize the importance of identifying the best therapeutic window to intervene in SMA.

\section{Therapeutic Window in Humans Based on the Natural History of the Disease}

In the first 6 months of life, infants with SMA type 1 (Werdnig-Hoffmann disease) show symptoms of proximal muscle weakness that affects the lower limbs more than the upper limbs [2].

The average age of death or the age when a patient starts to need more than $16 \mathrm{~h}$ per day of ventilation in SMA type 1 patients is 13.5 months [10]. A recent study of natural history of SMA showed that the median survival time for SMA infants excluding participants with more than two copies of SMN2 was 8 months [12].
The motor function decline, as determined by the Children's Hospital of Philadelphia Infant Test of Neuromuscular Disorders (CHOP-INTEND) scale in untreated children, showed a loss of 1.27 points per year. The highest score of the scale is 64 points; at disease onset, the mean score of SMA patients is 25 , and the maximum score is up to 40 points [10].

It has been demonstrated that infants with SMA type 1 undergo a rapid loss of motor units in the first 3 months of life, with more than $95 \%$ of motor units lost within 6 months of age [12, 13]. Denervation was determined using electrophysiological tests for the motor unit number estimation (MUNE) and maximum compound motor action potential amplitude (CMAP) studies [13]. SMA type 1 infants showed a uniformly low CMAP at the time of diagnosis, while in presymptomatic infants, the CMAP value was higher and decreased as patients became symptomatic [13]. In a case report described by Finkel, the CMAP value of an SMA type 1 patient who was identified while presymptomatic exhibited the same pattern of decline. The decline in the INTEND motor scale followed a parallel course to the decline in the CMAP, from presymptomatic at day 5 to moderately affected on day 34 . Further deterioration at day 63 , when the patient was fully symptomatic, was mainly observed in the patient's bulbar muscles and breathing functions, with motor function and CMAP values remaining at the nadir. The patient died at 81 days, and no autopsy was performed [10].

The extent of MN loss has been reported as profound in most postnatal autopsy studies. Soler-Botija et al. described an approximately $50-60 \%$ loss of MNs in one SMA newborn and four SMA infants aged 4-9 months of age [14]; moreover, Simic et al. described that approximately $73 \%$ of MNs were lost in five genetically confirmed SMA type I cases aged 5-22 months [15]. Simic et al. reported that $0.2-6.4 \%$ of the remaining $\mathrm{MNs}$ showed the activation of apoptosis (TUNEL positivity), while other studies did not report signs of apoptosis [14, 16]. Since apoptosis can be an ongoing, intermittent, and relatively rapid process, it may be very difficult to detect the occurrence of apoptosis at a single stage in an autoptic spinal cord report. It is also possible that apoptosis is more evident during the early phases of the disease [16] or that other mechanisms such as necroptosis occur.

To obtain beneficial results in treating severe SMA type 1 , the therapeutic window is narrow; based on neurophysiological and autoptic data, the period within the first 3 months appears to be permissive to have an impact on the disease phenotype, whereas the period between 3 and 6 months appears to be suboptimal for therapeutic intervention. However, therapies should be started well before the loss/damage of MNs, and the current results of the therapeutic trials suggest that the therapy should be administered prior to a month, and when is possible within a week after birth. 
Regarding the natural history of SMA types 2 and 3 [17], the motor functions tested with the Expanded Hammersmith Functional Motor Scale (HFMSE) from baseline presented a stable trend in the first year and a decline rate of 1.71 points in 3 years (having type $3 a$ resulted in a meaningful clinical difference). The ability to walk at 20 years of age for patients with SMA type 3 was $44 \%$ for SMA type 3a patients (onset < 3 years) and $89 \%$ for SMA type $3 b$ patients (onset $>3$ years) [17]. The therapeutic windows, based on neurophysiological and postmortem data, for SMA type 2 and 3 patients were less clearly defined [2]. In fact, studies including the correlation between presymptomatic and postsymptomatic electrophysiological data and postmortem data in SMA type 2 and 3 patients are limited [2].

To optimize the identification and treatment of the disease, newborn screening for SMA will allow for the early detection of candidates for approved therapy and for their enrollment in clinical trials. The therapeutic window determined in the clinical trial is discussed more extensively later in this manuscript. Furthermore, early diagnosis would accelerate the planning and the beginning of medical care for nutrition, respiratory, and physical therapy, thus preventing and curbing complications of the disease. The implementation of timely treatment would also reduce the cost of medical care. Moreover, newborn screening would prompt parents to be more prepared for genetic counseling in anticipation of a secondchild.

\section{Therapeutic Window in a Transgenic Model with Conditional Expression of SMN}

To discover and better understand the time-dependent role of SMN during MN development and maturation, different SMA animal models were generated (Fig. 1).

In the zebrafish (Danio rerio) SMA model, the absence of SMN compromised dendritic and axonal growth [18]. The requirement for SMN was greatest during embryogenesis, when MNs establish connections with their respective targets [18]. The period within $36 \mathrm{~h}$ postfertilization corresponds to the development of the central nervous system and other organs of the embryo, and no benefit was observed when SMN protein was added after this period (Fig. 1). It is important to notice that whilezebrafish show developmental abnormalities of motor neurons, this finding does not appear to be replicated in the mouse models $[19,20]$.

Le et al. developed an SMA mouse model in which the SMN2 expression was inducible only by the administration of doxycycline; in this study, the protein expression was induced at different time points. Le et al. found that in the embryonic or early postnatal period (0 and 1 postnatal day (PND)), the induction of SMN2 expression resulted in a substantial rescue of SMA mice (survival over 200 days), while postnatal induction in later periods resulted in reduced rescue. The critical window of intervention was between birth and PND4 (Fig. 1). Delaying SMN induction by only 1 day, with SMN expression detected at PND5, diminished the benefits in terms of the survival duration and activity of SMA mice.

Furthermore, the long-term requirement of SMN was investigated by withdrawal of doxycycline at PND28 in rescued mice. One month after the removal of doxycycline, the mice mostly demonstrated a rapid decline in motor functions and died at 1 month of age, despite the observation that there were no differences in neuromuscular transmission compared to heterozygous controls. This result suggested that the function of the neuromuscular junction (NMJ) was not dependent on the expression of SMN in adult mice and that two copies of SMN2 are sufficient for survival in adults but not during the critical postnatal period [21].

An independent study investigated the effect of inducing SMN expression in SMA mice using a tamoxifen-inducible CRE-responsive allele [22]. The earlier the protein was restored, the fewer abnormalities of NMJs were observed, and consequently, the more effective the therapeutic intervention was deemed to be; these results revealed that there was a narrow temporal sensitivity of transgenic mice MNs placed between PND4 and PND8 (Fig. 1). This work confirmed that reactivation of SMN protein after disease onset could halt the
Fig. 1 Schematic representation of the therapeutic window identified in animal models of SMA type 1 . Along the lifespan of the animals, the colors represent the period when the therapy can take effect: pink is the period when the therapy can be most effective, while orange and yellow are the age ranges for which the administration of the therapy has progressively less efficacy.h hours after fertilization, B birth, PND postnatal day

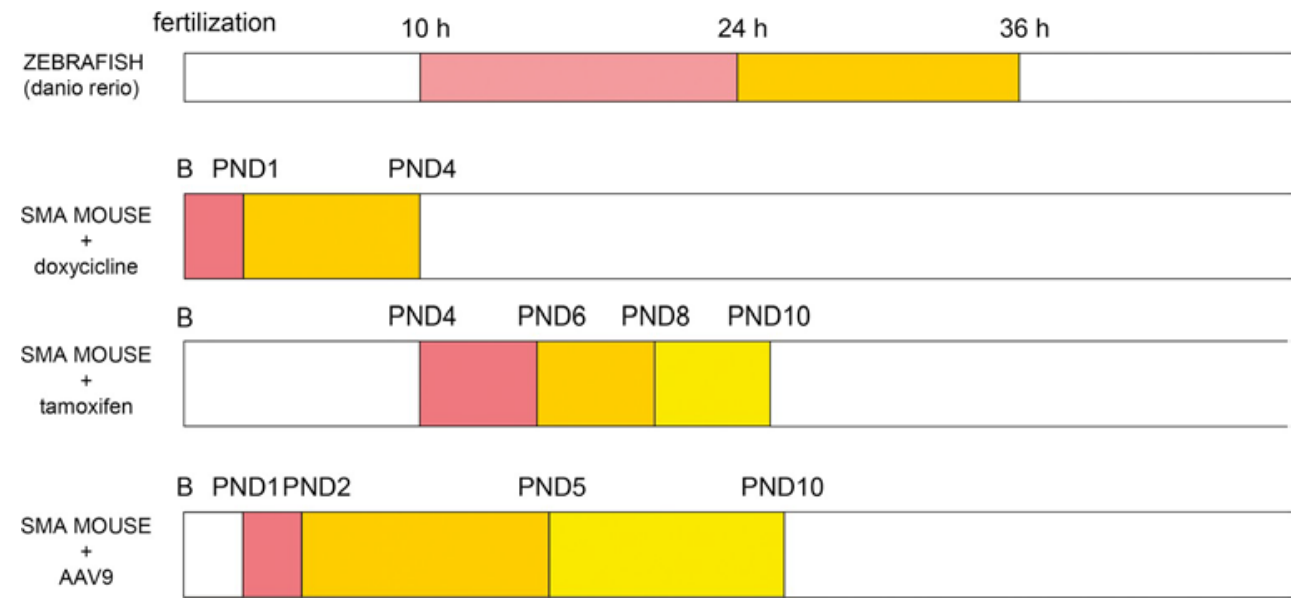


progression of SMA pathology, and thus, earlier intervention (i.e., prenatal) was required to reverse the deficit in overall growth.

Nevertheless, it is critical to intervene in SMA before MN loss has occurred because SMN expression cannot be effective once neurons have died.

Since direct SMN restoration represents a therapeutic option in SMA, it seems important to define, in addition to than the optimal window of opportunity for therapeutic intervention, whether the requirement of the protein changes with time and/or is dependent on other factors. A line of tamoxifen-responsive SMN2 transgenic mice was used to investigate this purpose. Neonatal depletion of SMN caused the development of a severe and fatal SMA phenotype. Similar results were observed when SMN was depleted at different time points between PND4 and PND12, such that mice exhibited rapid loss of body weight and impaired motor abilities that resulted in muscle paralysis [23]. In contrast, administration of tamoxifen and consequent SMN depletion at PND15 lessened the severity of the disease, as if a reduced quantity of the protein was required for normal neuromuscular function in adult age. The transition from an SMN-dependent state to a state of relative SMN insensitivity was identified as a 3-day window between approximately P12 and P15, which reflected the period of transformation of NMJs into mature synapses. An insufficient amount of SMN protein impaired longterm maintenance of MNs and muscles, resulting in the eventual alteration of NMJ and muscle fiber pathology. Indeed, these structures need to be replaced periodically during adulthood as a consequence of the normal process of aging, injury, or disease. Further experiments demonstrated that inducing NMJ turnover by injuring the nerve cells aroused the requirement for high levels of SMN protein. This was also true for muscle regeneration, highlighting the critical role of SMN in the regulation and maintenance of the neuromuscular system. Experiments conducted in SMA mice showed a correlation between the timing of SMN restoration and the development of plaque-like AChR (acetylcholine receptor) clusters [24]. This result indicated an important role of the SMN protein in the maintenance of MNs and in the formation of adult neuromuscular synapses.

The NMJ maturation and regeneration in response to internal or external factors defined the temporal requirement for the SMN protein. If so, a therapeutic SMNenhancing agent in human patients could be administered in an early postnatal period, then tapered during adulthood once the neuromuscular system has fully developed and then reintroduced as needed in response to injuries or aging. However, this can be true for drugs modulating $S M N$ gene activation and alternate splicing or stabilizing SMN protein (e.g., small molecules, antisense oligonucleotides
(ASOs), and other drugs), nor for gene replacement strategy (i.e., gene therapy).

The neuronal aspect and the phenotype of the animals were influenced and corrected after cellular pathology evidence and symptom onset. These results extended the therapeutic window, and provided the rationale to treat young patients, also in postsymptomatic period, by modulating the expression of the SMN2 gene or delivering the SMN1 gene [22].

Nevertheless, it should be noted that SMA mouse models differ from human patients since they show peripheral organ deficiency and malfunction (e.g., significant reduction in spleen size, intestinal and metabolic abnormalities) compared to SMA patients. Such abnormalities are lesser or not at all described in human beings, who developed most of all a neurologic phenotype. Therefore, $\mathrm{MN}$ involvement seems to be stronger for human patients than mouse models, suggesting a greater SMN sensitivity also in adult age.

\section{Milder Models of Disease}

Studies describing the severe phenotype mouse models of SMA, with a median survival of 12-14 days after birth, represent the principal source of knowledge about the temporal requirement for SMN in mammals. Nevertheless, the rapid disease progression of these mice, combined with some phenotypes, such as cardiac defects, limited their use in testing the efficacy of therapeutic interventions. Milder models of SMA have been generated by Bogdanik et al. in order to assess the temporal window for therapeutic SMN restoration in SMA type $2 / 3$ patients [25]. The $\mathrm{BBurgheron} \wedge$ mouse exhibited an intermediate lifespan (mean survival of 89 days), delayed structural maturation of NMJs, and specific electrophysiological findings (reduction of CMAP and MUNE). Systemic administration of ASOs has been found to restore neurological phenotype and to extend mouse survival. In severe SMA mice, SMN restoration was efficacious within the early postnatal days, and in milder models, positive effect of intervention was also obtained after the onset of neurological symptoms.

Zhou et al. generated an intermediate SMA model by administering a low-dose morpholino antisense oligomer PMO25 to neonatal mice from a Taiwanese model of severe SMA [26]. PMO25 was administered at different time points and with different modalities: periodic systemic administration of low dose of PMO25 (at 2-week intervals) showed longer term therapeutic benefits than single low-dose (PDN5) and single bolus high-dose injection at PND0 (with better results when the bolus was administered earlier in the disease course).

Another recent study succeeded in obtaining a milder SMA phenotype through administration of a suboptimal dose of SMN2 splicing modifier in a $\Delta 7$ mouse model [27]. These mice survived longer (reaching adult age) than untreated mice 
and showed adult SMA-like pathology, i.e., synaptic defects in NMJs and muscle atrophy. After manifestations of symptoms, the administration of an increased dose of the splicing modifier ameliorated the synaptic alterations, and the administration of a myostatin inhibitor reduced atrophy of muscle fibers in $\Delta 7$ mice that obtained a suboptimal dose. Thus, this work provides a murine model of mild SMA pathology, resembling the phenotype of adolescent and adult patients. In addition, these experiments suggested that the window of intervention in SMA types 3 and 4 seemed to be linked to age of onset of the disease and might be broader than types 1 and 2 . Thus, SMN-targeted therapies administered after disease onset may only have a limited capacity to improve disease phenotype. In contrast, rescue of SMN levels in a milder SMA mouse model significantly ameliorated motor abilities, pointing out a potential difference related to the timing and nature of the therapeutic window in severe versus mild forms of SMA.

\section{Therapeutic Window in SMA Mice Preclinical Therapeutic Experiments}

Since the loss of MNs and neuromuscular symptoms in SMA are due to a genetic defect in the gene coding for SMN protein, one of the most promising therapeutic interventions is the delivery of the wild-type allele to affected neurons. Several studies have been performed to address SMN gene delivery to target cells through viral vectors; in these studies, bypassing the blood-brain barrier (BBB) to reach the central nervous system was one of the most challenging issues. During development, the permeability of the BBB progressively decreases due to the growth of the capillary endothelial cells. Furthermore, it is noteworthy that, in SMA pathogenesis, the younger the subject is, the healthier the MNs are. It is therefore crucial to identify the optimal temporal window in order to enable the vector to reach the correct target and the majority of cells.

Theintravenous administration of adeno-associated virus 9 (AAV9) was indeed tested in mice at different ages [28]. In neonatal mice, systemic delivery of the AAV9-GFP fluorescent reporter resulted in a robust expression of GFP in the heart, skeletal muscles, and spinal cord with transduction in the dorsal root ganglia, in the MNs of the ventral region (56 and $61 \%$ of MNs were positive for GFP 10 and 21 days after injection, respectively), and in astrocytes. Neurons and astrocytes in the brain were also positive for GFP expression. When the same vector was administered to older mice, histochemical analysis revealed a loss of transduction in the dorsal root ganglia and a marked decrease in the GFP-positive MNs in the ventral part of the spinal cord. However, GFP-positive astrocytes were found at all levels of the spinal cord, and selective neuronal expression in the hippocampus and dentate gyrus was also observed.
These data demonstrated that there is a crucial period during which the cells of the dorsal root ganglia and the lower MNs can been reached from the circulatory system. The predominant astrocyte transduction in adults suggested that the viral construct escaped the brain vasculature. Gray et al. found that the transduction of GFP throughout the brain and spinal cord was dose-related with neurons outnumbering astrocytes in the hippocampus (2:1) and striatum (1.9:1) but not the cortex (1:1). Structures outside of the BBB showed higher overall transduction. AAV9 transduced both neurons and astrocytes. Biodistribution of GFP was also dose related in the kidneys, while in the other tissues (liver, spleen, lung, and skeletal muscle), there was a possible saturation of transduction capability of AAV9 at a medium or high dose [23]. The following step was to test the injection of self-complementary (sc) AAV9 carrying the full-length $S M N$ cDNA gene in the facial vein of 1-day-old SMA mice.

The treated mice exhibited an improvement in the motor functions, were larger in size, and had higher body weight compared to untreated mice. The lifespan increased to at least 250 days [29]. Based on this study, scAAV9-SMN was injected at different time points. In mice treated at P2, western blot analysis showed elevated SMN expression in the brain, spinal cord, and muscles, despite not reaching the same level of expression as in wild-type mice. Interestingly, delaying treatment at P5 determined the occurrence of partial rescue of the disease with a reduced lifespan. Finally, injection at P10 had little or no effect on reverting the pathological phenotype, suggesting once again the importance of early treatment to achieve the maximal benefit [29] (Fig. 1). Passini et al. investigated the distribution of the SMN transduction according to different amounts of sAAV9-SMN delivered into the CNS (through cerebral lateral ventricles and lumbar spinal cord) of SMA mice at 1 postnatal day. They found that the SMN levels correlated with the doses, and the cells expressing the protein were mostly MNs localized to the lumbar region of the spinal cord. The percentage of positive MNs decreased in the regions that were more distally positioned from lumbar injection site. A greater number of positive MNs were found in the lumbar, thoracic, and cervical regions of mice treated with higher dosages of SMN. It was also observed that the average lifespan increased according to the dose used, and the mice receiving the highest dosages showed consistent weight gain [30].

\section{AAV9 Administration Testing in Large Animals}

Since CNS development is different in mice and primates, the timing and efficiency of MN transduction was investigated through scAAV9 administration in non-human primates. As there is no primate model of disease, the vector has been labeled with the GFP. ScAAV9-GFP was intravenously injected into a healthy male cynomolgus macaque on P1. 
After 25 days, GFP-positive cells were detected in dorsal root ganglia and MNs of the entire neuraxis, demonstrating the ability of the vector to cross the BBB in young non-human primates [29]. The transgene expression was then explored in non-human primates at different time points from birth through 3 years of age [31]. The expression was similar to that found in rodents. In all monkeys treated through intravascular delivery at 1,30 , and 90 postnatal days, the GFP positivity was seen primarily in neurons, especially in large MNs of ventral horn, and in some glial cells scattered throughout the spinal cord. Conversely, in the brain, microglia and astrocytes were the most prominent cell types targeted. In addition, GFP-expressing cells were seen in all cortical regions, lateral geniculate, midbrain, pons and medulla, thalamus, and putamen. The intravascular delivery of the vector in a 3-year-old macaque determined a widespread GFP expression in the brain, with cortical and pontine regions having the highest prevalence of transduced cells. Systemic gene delivery also targeted multiple peripheral organs (skeletal muscles, liver, medulla, spleen, testes, lungs, and kidney) except for the heart, where the transduction was not efficient [31].

In contrast with experiments in mice, the finding that gene delivery in a young non-human primate ( 3 years old) can preserve SMN gene expression in not only glia or astrocytes but also in MNs could suggest that this approach is applicable in humans and is useful for the treatment of SMA types 2 and 3 [31]. To further increase the potential role of gene therapy, direct CSF delivery has been speculated. The intrathecal vector delivery resulted in a spread distribution of GFP in the brain (from the prefrontal to the occipital cortex and in the cerebellum) and the spinal cord similar to systemic delivery; however, the number and the intensity of GFP-positive cells were greater (10-fold). GFP expression was observed with MN transduction of 25-75\% in all the segments. The largest number of transduced cells was found in the cerebellum. In the cortex, GFP positivity was found primarily in pyramidal cells and in astrocytes [30,32-34]. In the animals that received a higher dose, the number of positive MNs was higher, and transduction was clearly detected also in the glial and neuronal cells of the brain cortex and in the Purkinje cells [30]. Moreover, the intrathecal administration implied a lower periphery distribution of the AAV9 [32]. After viral injection through a lumbar puncture in a 1-year-old cynomolgus macaque, significant GFP transduction in the spinal cord was obtained in 29,53 , and $73 \%$ of the cervical, thoracic, and lumbar regions, respectively. The transduction rate with the same dose seemed to improve when subjects were kept in the Trendelenburg position for $10 \mathrm{~min}$ after vector infusion (targeting 55, 62, and $80 \%$ of MNs in the three regions of the spinal cord, respectively) [35]. The presence in the host animals of the antibodies against AAV9 nullified the vector and the intended function. In non-human primates that presented preexisting AAV9 antibodies, it was reported that no vector genome was detected after $24 \mathrm{~h}$ [23] when treated intravenously. Conversely, in the monkeys that were treated with CSF delivery, no difference was found in the distribution between subjects with and without preinjected antibodies against the vector [27]. The window of opportunity to target spinal MNsinnon-human primates encouraged the translation to newly diagnosed SMA children. The intrathecal delivery of the AAV9-GFP was also tested in 2-month-old pigs, and GFP expression in $10-30 \%$ of the MNs in the lumbar region occurred in a dose-dependent manner [36]. Intrathecal and/or intracisternal injection of AAV9-GFP in 5-day-old pigs resulted in gene expression in MNs at all levels of the spinal cord, and the cerebellar Purkinje cells, nerve fibers, medullar nuclei, and scattered cells near the meningeal surface were also easily targeted.GFP expression in peripheral tissue was not detected, suggesting that the virus was localized mostly in the CNS [31]. A large SMA model in the domestic pig was created by intrathecal delivery of scAAV9 expressing a shRNA targeting pig SMN. A SMA-like phenotype was obtained reducing the SMN mRNA levels by $73 \%$ in MNs postnatally. CMAP and MUNE measures presented a correlation with the induced phenotype progression in these animals, similar to SMA patients. After that, restoration of SMN through scAAV9 expressing human SMN administration was tested at different time points. When the second treatment was administered at symptoms onset and before CMAP and MUNE changes, the phenotype course resulted affected. Also when the scAAV9-SMN was delivered after symptoms onset, the improvement of the neuropathology and of neurophysiologic parameters (CMAP and MUNE) was observed, pointing out the possibility to intervene later in the disease process altering the progression of symptoms. The improvement of CMAP value at later time point it has been hypnotized relates to collateral re-innervation [37].

There are differences in the response of each species to gene treatment, in comparison to mouse model where astrocytes have widespread expression of the SMN protein; in pig only, rarely glial cells are transduced; furthermore, in the murine model, the distribution in the periphery seems to be relevant for the survival of the animal. These difference are likely related to a peculiar species intron structure that influence the splicing of genes in relation to the SMN levels, or a different sensibility to the human SMN2 regulatory regions [37].

\section{Therapeutic Window in Clinical Trials}

The first approved drug for SMA is Nusinersen (IONISSMNrx), which is a 2'-O-methoxyethyl phosphorothioatemodified antisense drug designed to bind the SMN2 premRNA and to promote the inclusion of exon 7. Nusinersen was first tested in an open-label, single-ascending dose study in patients with symptomatic SMA aged 2-14 years [25]. Patients who received the lowest doses $(1,3$, or $6 \mathrm{mg})$ did 
not experience a significant change in motor performance, while the 9-mg group exhibited a mean increase of 3.1 points in the HFMSE scores from baseline at day 85. Moreover, 7 out of 10 participants (70\%) exhibited an increase of 3 to 7 points. The improvement was observed in both SMA type 2 and type 3 patients, in five out of seven and in two out of three subjects, respectively, and across the age range with three children aged more than 5 years and four children aged less than 5 years. At the long-term follow-up, the mean change in HFMSE score from baseline to 9 to 14 months later was 5.8 points. Six of eight participants had an increase greater than 3 points. No participants in the 9-mg cohort declined in HFMSE score, and the range of improvement was 1 to 14 points [38]. Patients with SMA type 1 enrolled in the phase 2 open-label study of Nusinersen showed important improvement in motor function measures, i.e., they acquired the ability to maintain the upright position and to walk independently [39]. Included patients were aged between 3 weeks and 7 months old, with the onset of spinal muscular atrophy symptoms between 3 weeks and 6 months. A promising clinical response to Nusinersen in most, but not all, infants with spinal muscular atrophy was observed; however, no comment specifically proposed that this failure could have been correlated with later treatment. Postmortem analysis of patients treated with Nusinersen showed drug uptake into MNs throughout the spinal cord and in neurons and other cell types in the brainstem and other brain regions. Moreover, in the spinal cord, SMN2 mRNA exon 7 inclusion was upregulated and the SMN protein level was increased. The interim analyses performed during the phase 3 clinical trial, in which infants with SMA type 1 (ENDEAR) and children with SMA type 2 (CHERISH) were enrolled, showed a good safety profile and the achievement of primary endpoints for motor performances (Chiriboga et al. 2017). In the interim analysis of the ENDEAR study, a significantly higher fraction of SMA patients in the Nusinersen group than in the control group had a motor milestone response ( 41 vs. $0 \%, P<0.001$ ), and this result prompted to an early termination of the trial [40]. In the final analysis, a significantly higher proportion of SMA patients in the Nusinersen group than in the control group had a motor milestone response ( 51 vs. $0 \%$ ). However, of the infants who presented motor milestone response, only $8 \%$ could sit independently and $1 \%$ could stand. By the end-of-trial cutoff date, $39 \%$ of the infants in the Nusinersen group and $68 \%$ in the control group had died or received permanent assisted ventilation [40]. It is important to notice that the best results of ENDEAR study were observed in patients who started treatment within 13 weeks after the onset of disease [40].

Presymptomatic treatment might provide an even greater clinical response; this hypothesis is being examined in an ongoing phase 2 study (Nurture, NCT02386553). Data presented at a meeting in April 2017 showed that presymptomatic treatment in SMA type 1 patients (treated within 6 weeks of age) is likely more beneficial than in the symptomatic cohort tested in the open label and ENDEAR studies (Chiriboga et al. 2017).

Overall, the data obtained with the Nusinsersen trials suggest that in SMA type 1, treatment within 3 months is associated with some motor milestone response, but to have a satisfactory clinical meaningful effect treatment within 1 month, or even fewer weeks, is probably required.

The favorable data obtained during the abovementioned trials led to the worldwide approval of Nusinersen treatment for SMA type 1 patients and for treating SMA types 2 and 3, first by the Food and Drug Administration in the USA and more recently by the European Medical Agency in Europe.

Useful information about the therapeutic window in humans came from the first gene therapy trial in SMA patients.

Based on extensive preclinical efficacy and safety studies in mice and non-human primates, in May 2014, the Phase 1/2 BGene Transfer Clinical Trial for Spinal Muscular Atrophy Type $1^{\wedge}$ (clinicaltrials.gov identifier NCT02122952) was designed. Patients with SMA type 1 carrying biallelic SMN1 mutations and two copies of SMN2 were enrolled. Gene transfer was obtained via intravenous infusion of a scAAV9 carrying the SMN gene (AVXS-101) under the control of a hybrid CMV enhancer/chicken- $\beta$-actin promoter. Depending on the cohort, SMA type I patients were 6 or 9 months of age and younger (mean age 3.4 months in higher dose cohort). Cohort 1 (lower dose $6.7 \times 1033 \mathrm{vg} / \mathrm{kg}$ ) included three subjects, and cohort 2 (higher dose $2.0 \times 109 \mathrm{vg} / \mathrm{kg}$ ) enrolled 12 children.

The published data showed a favorable safety and welltolerated profile of AVXS-101 and, furthermore, the patients' achievement in motor development milestones. In both the cohorts, there was an increase in the CHOPINTEND score and in the achievement of motor milestones. In particular, of the 12 patients in the high dose cohort, 11 were able to sit without support, 9 to roll over, 11 to feed orally and to speak, and 2 to walk independently. The study was closed in January 2017, and the data were published on November 2017. No new events were reported, and 15 of 15 patients were event-free at 20 months of age; the efficacy results were encouraging. It appeared that the children treated with the proposed therapeutic dose early in age (in the first month of life) and early in the disease course achieved more advanced motor milestones and did so more rapidly, emphasizing the need for newborn screening in SMA1 when treatments become available [41]. Overall, the data obtained with the gene therapy trial also suggest that in SMA type 1, treatment within 3 months is associated with significant motor milestone response, but to have a greater clinical effect (i.e., to achieve independent walking) treatment within 1 month, or even within fewer weeks, is likely needed. 


\section{Discussion}

\section{Pathophysiology of the Disease and the Therapeutic Window}

Understanding what limits the therapeutic time window may be crucial to implementing efficient combinational therapeutics, which can increase the efficacy of SMN-dependent therapies. SMA, in its most severe forms, presents a clinical onset during the perinatal period that is a crucial time of neuromuscular development and maturation. Thus, key aspects in SMA pathogenesis involve defective motor neuron development and progressive loss of motor neurons. It is not clear whether there exist elements that limit this therapeutic time window during very early stages in disease, and if so, what these elements are. One hypothesis is that this time can correlate with irreversible motor neuron damage, beyond which even restoring SMN levels is not sufficient for their survival. This time point can also reflect a developmental requirement for SMN within the neuromuscular system, in particular in severe types of SMA, which may limit the efficacy of SMN replacement therapies.

Some studies have revealed that motor neuron and synapse defects are present during fetal development, supporting a role for SMN in motor neuron development [16]. This can be more prominent in the case of severe reduction of SMN. However, these developmental aspects might be compatible with a full functional rescue, as indirectly proven by the preliminary results of a full rescue of subjects treated during the presymptomatic stage (Nurture trial). Indeed, this study also suggested that early rescue of SMN level can halt the motor neuron degeneration. The two elements to reduce the impact of the therapeutic window for an SMN-dependent strategy to preserve motor neurons are represented by increasing the efficacy and biodistribution of gene therapy/ASO treatments for SMA to allow a maximal and rapid rescue of SMN and by implement SMA neonatal genetic screening for presymptomatic treatments.

The first sign of motor neuron distress is axonal withdrawal and neuromuscular junction impairment. When the soma of the motor neuron is healed through the increase in SMN expression, it can regrow the axon and restore a proper neuromuscular junction connection.

However, nerves that have sustained injuries closer to their soma are less likely to survive and regenerate, and chronic denervation is exacerbated by the slow axon regeneration rate of $\sim 1 \mathrm{~mm}$ /day and axon misdirection, where axons reconnect to the wrong target [42]. Strategies that promote proper axon regrowth are important but still underdeveloped in motor neuron diseases.

Proper development and organization at the NMJ are necessary for effective neuromuscular transmission. However, SMN reduction may affect the axon innervation and the organization/maturity of the acetylcholine receptor, resulting in the impairment of neuromuscular transmission. It has been hypothesized that an alteration to normal developmental processes could directly contribute to NMJ pathogenesis in SMA patients. This led to the hypothesis that reduced levels of SMN could alter the development of some, or all, cells contributing to the NMJ, thus causing this structure to be selectively vulnerable to subsequent degeneration during disease.

In theory, injured peripheral nerves and skeletal muscles have a natural ability for tissue regeneration [43], and regeneration of functional NMJs occurs after NMJ injury [44]; thus, when SMN is restored, NMJ and muscles can recover. However, this process is very delicate. In fact, the alteration of regenerated NMJs formed at both original synaptic sites and new synaptic areas can be associated with permanent neurological deficiency and long-term skeletal muscle contractile dysfunction. It is unknown whether these aspects can influence the recovery of SMA after treatment. NMJ can be an important target for expanding the therapeutic window. Restoring SMN protein in SMA mice during early postnatal life resulted in the accumulation of presynaptic and postsynaptic abnormalities of the neuromuscular junction of mutant mice, which eventually precluded any benefit that could be obtained from restoring SMN [22]. Progressively fewer defects were observed the earlier the protein was restored.

Future therapeutic strategies should be focused on extending the therapeutic time window, in particular by stabilizing the neuromuscular system for a longer period. This would allow for a greater therapeutic benefit from SMN-targeted therapies (such as oligonucleotides or gene therapy), and/or would independently act to stabilize the neuromuscular system beyond the time window that currently is present for SMN-targeted therapies. In this sense, the investigator compound Olesoxime acts as neuro-protectant on motor neurons, preventing excessive permeability of the mitochondrial membrane under stress conditions [45].

Muscular activators such as CK-2127107 (CK-107) can improve muscle function of SMA patients by enhancing muscle contractions. CK-107 is a fast skeletal muscle troponin activator and is intended to slow the rate of calcium release from the regulatory troponin complex of fast skeletal muscle fibers and thus improve muscle function. While this type of strategy did not require a specific time window, being SMNindependent, it required at least some degree of muscle functionality.

It is important to identify novel molecular with therapeutic target independent from SMN and acting synergistically on therapies that restore the protein synthesis.

\section{Conclusions and Perspectives}

SMA is a neurodegenerative disease in which MN degeneration and loss are continuous and progressive from birth. The 
general assumption is that the sooner the therapy is started, the greater the advantage will be. A precise therapeutic window has not been defined. In SMA type 1, according to the experimental data obtained so far, the therapeutic window can be hypothesized to be, optimally, within 1 month of age or even within few weeks after birth (ideally 1 week), sufficient to have a motor function response within 3 months of age and, well suboptimally, within 6 months (Fig. 2). The therapeutic timeframe for SMA type 2 to 4 is undefined. All patients, until now, enrolled in clinical trials with oligonucleotides and gene therapy were children under the age of 12 years.
Therapy should be applied when MNs are still alive and should aim to preserve SMN function during cell development and during elaboration of neuromuscular synapses [22, 49]. The clinically detectable motor impairment can be more prominent than the number of soma of motor neurons lost since instability of NMJ or distal axonal loss is sufficient to result in muscle weakness. Thus, it can be hypothesized that in the early symptomatic stage, even if hyposthenia is evident, a prompt restoration of SMN level could allow motor neurons to regrow these axons and recreate their connections with muscles.

a

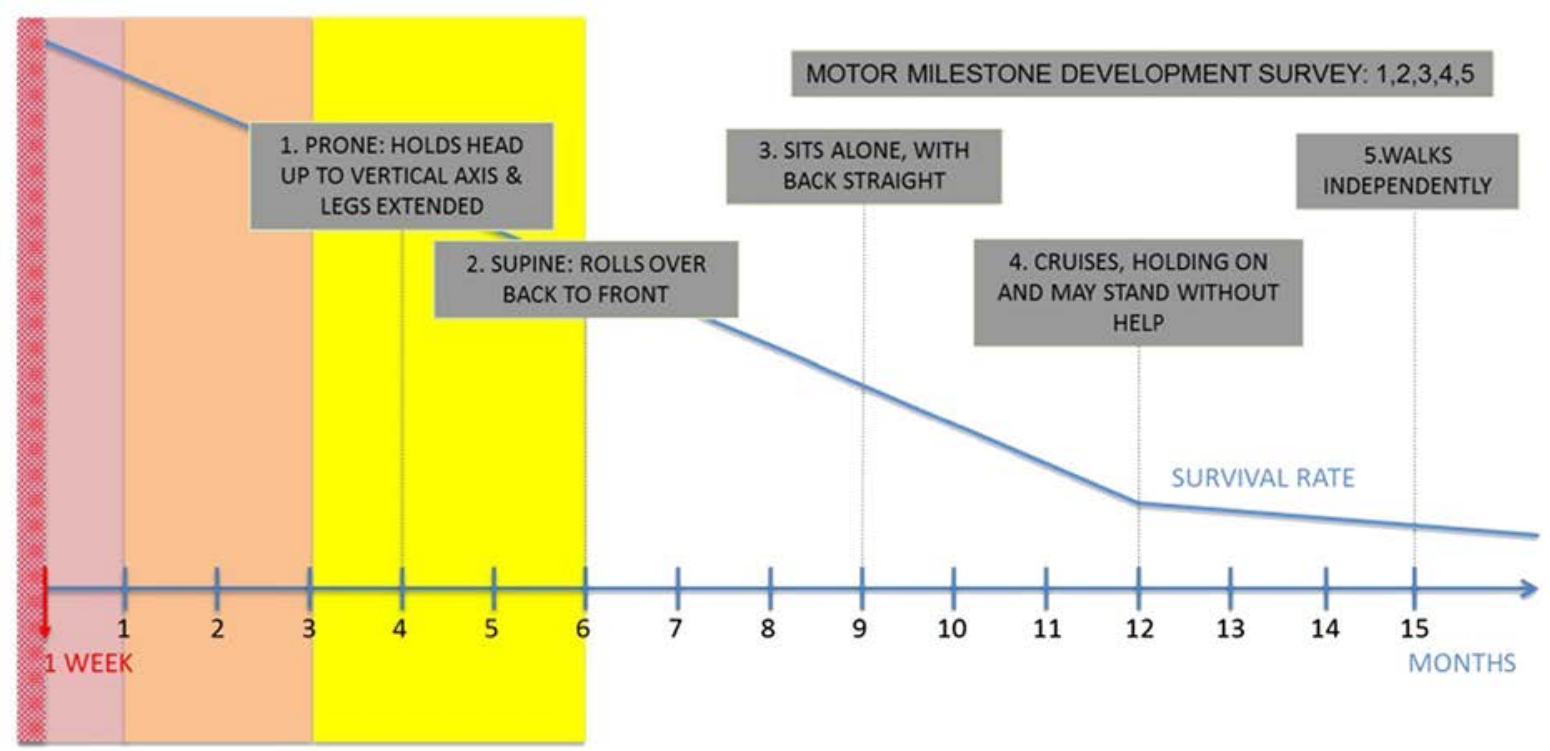

b

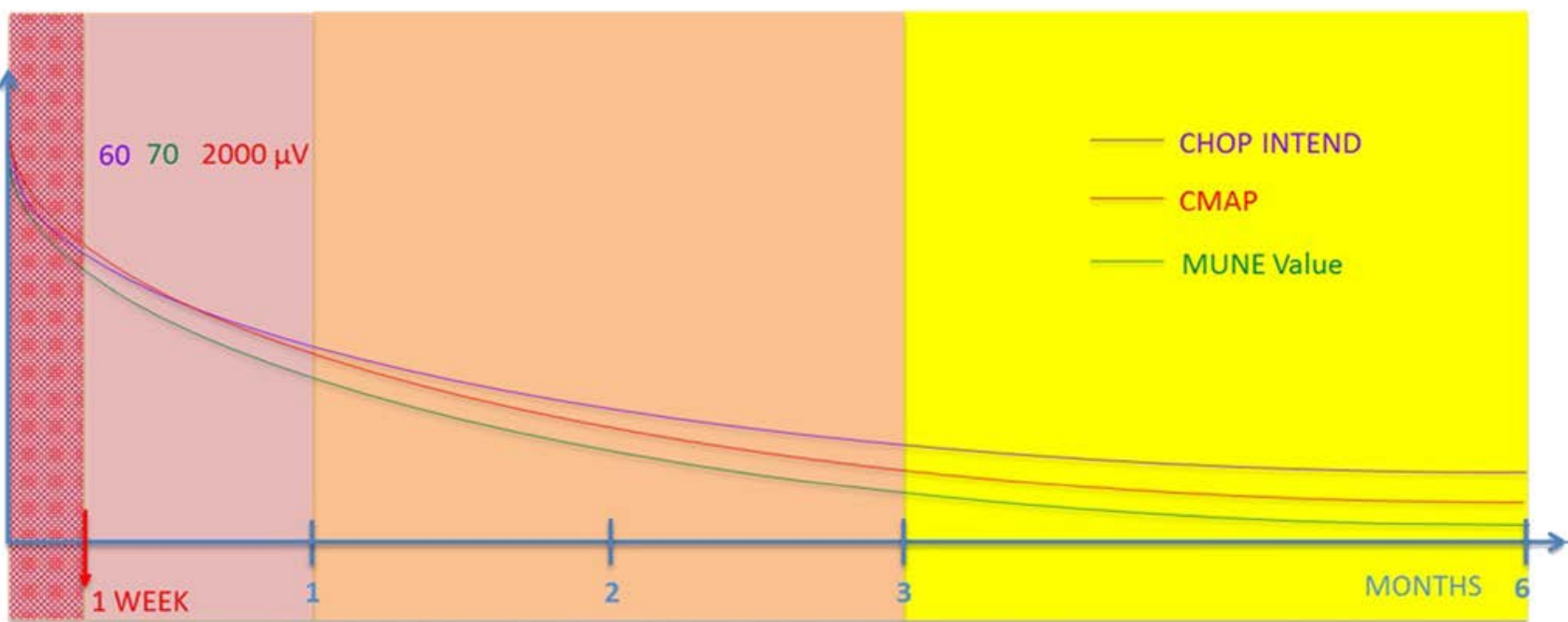

Fig. 2 Therapeutic window in humans. a For patients with SMA type 1, the mortality rate is 25 to $50 \%$ by 8 months and 75 to $90 \%$ by 12 months of age (Niranjan et al. [46]; Bach et al. [47]; Finkel et al. [48]). Gray represents the motor milestone for healthy subjects. b The violet, red, and green curves represent the CHOP INTEND, CMAP, and MUNE trends, respectively, in SMA type I patients. Patients treated with SMN rescue therapy at less than 1 month of age developed motor performance comparable to that of healthy subjects. According to the residual motor unit estimates with the CMAP and MUNE motor performances and the amelioration obtained in clinical trials so far, the best period for therapeutic intervention was within 1 month (pink area), and ideally within 1 week after birth (red net area). Additionally, when the treatment was administered in the following months, a gain in motor performances was observed to be higher within 3 months (orange area) and milder later in life (yellow area) 
Experiments conducted in different animal models of the disease showed different timing for intervention of SMN protein in the development and maintenance of MNs, in particular their distal compartment (axon and NMJ). The requirement of SMN protein during the embryonic and early postnatal period is consistent with the early age of onset of SMA in human patients.

SMN-enhancing agents were most effective in halting the progression of SMA if administered early on during the disease before complete maturation of neuromuscular synapses has been reached. Furthermore, tapering of drug concentrations and possible subsequent reintroduction should be considered once the disease has been stabilized. However, it seems that SMN represents a maintenance factor for NMJ structure and is invoked at specific moments of adult life. Thus, SMN can also be required during muscle regeneration in adulthood.

Shortly after birth, the blood-brain barrier is relatively permeable and more easily crossed by therapies; moreover, the probability that younger patients will present immune response against viral vector is lower. Although a specific window of opportunity has not yet been defined in humans, it seems reasonable to postulate that there is a short critical period after which therapeutic advantages appear to wane. Moreover, in the severe SMA phenotype, this crucial window is narrow and short-lived.

The maturation processes and the modifications of CNS structures (BBB, neurons, and extracellular matrix) after birth and during infancy can have an impact on the drug (AAV and oligonucleotides) biodistribution and, consequently, on the therapeutic window. The distribution of the drug through the tissues to the target cells could be implemented using molecular strategies as peptide conjugation for oligonucleotides. SMN restoration may not only arrest but also reverse an overt disease phenotype, with important therapeutic implications for human beings. There is evidence that earlier intervention is more effective at modulating disease severity than intervention at later stages, resulting in longer survival and better motor performance, although there are mild benefits even when SMN protein is restored at relatively later stages. Currently, ongoing clinical trials are based on the use of gene therapy with AAV9 to deliver the SMN1 gene and on the use molecules that modulate SMN2 splicing. So far, data provided have shown promising results. The rapidity and the efficacy with which results are achieved in clinical trials might accelerate the procedures for approval by healthcare organizations of the experimental drug as therapy for SMA.

Looking at the results presented so far by the scientists promoting clinical trials with antisense oligonucleotides (Nusinersen) and gene therapy (AAV9-SMN), patients with SMA type 1 treated at an earlier age achieved the best outcome measure results (Fig. 2). A few studies showed that soon after birth, patients with SMA type 1 presented low CMAP and MUNE levels that rapidly decreased in the first few months of life [10, 12, 30, 50]. It seems reasonable to hypothesize that the first 2 to 3 months are the limit within which the therapy administration can potentially impact the SMA1 disease phenotype. Nevertheless, it seems that the treatment is more effective when given at a higher dose and at an earlier time point.

Prompt administration of SMN-restoring agents (within days from diagnosis), together with routine newborn screening for SMA, will optimize results in clinical trials. Positive results and the deeper understanding of the functions of new molecules suggest that these molecules could also be applicable for other genetic diseases. In particular, gene therapy would be ideal for treatment of spinal muscular atrophy with respiratory distress type 1 (SMARD1); SMARD1 is a neurodegenerative disease caused by mutations in the IGHMBP2 gene, which begins in childhood and leads to inexorable muscular atrophy. Encouraging results have been obtained in preclinical studies for this disorder; in fact, the phenotype of a SMARD1 mouse model was rescued after systemic injection of an AAV9 construct encoding the wild-type IGHMBP2 to replace the defective gene [51].

For both SMA and SMARD1, early treatment is essential despite the fact that the therapeutic window for both of these diseases in humans has yet to be established. The different molecules currently under evaluation are being shown to be excellent therapeutic possibilities to treat SMA. It becomes essential to realize and finalize the clinical trials in order to compare the safety and efficacy profiles of each molecule to the others and optimize their use in accordance to the patient clinical features. The timing of administration is of crucial importance and is closely linked to the time of diagnosis. Time is motor neuron in infantile SMA, a finding that reflects the neurological urgency of treatment. Therefore, in parallel with the optimization of therapy, it is necessary to implement a neonatal screening program with the future prospect of possible early intervention or prenatal diagnosis.

Acknowledgments We gratefully acknowledge the Joint Program Neurodegenerative Disease (JPND) Research grant DAMNDPATHS (2014) awarded to SC and the Ginevra SMARD1 fund. We thank the Associazione Amici del Centro Dino Ferrari for its support.

\section{References}

1. Faravelli I, Nizzardo M, Comi GP, Corti S (2015) Spinal muscular atrophy-recent therapeuticadvances foranoldchallenge. Nat Rev Neurol 11(6):351-359. https://doi.org/10.1038/nrneurol.2015.77

2. Sumner C, Paushkin S, Ko CP. Spinal muscular atrophy disease mechanisms and therapy. 1st Edition ISBN: 9780128036853 Academic Press 4th November 2016, Spinal Muscular Atrophy: 125 Years Later and on the Verge of a Cure M. Oskoui1, B.T. Darras2, D.C. De Vivo3 cap 1, 3-17 
3. Monani UR (2005) Spinal muscular atrophy: a deficiency in a ubiquitous protein; a motor neuron-specific disease. Neuron 48(6):885-896. Review. https://doi.org/10.1016/j.neuron.2005.12. 001

4. Pearn J(1978) Incidence, prevalence, and genefrequencystudies of chronic childhood spinal muscular atrophy. J Med Genet 15(6): 409-413. https://doi.org/10.1136/jmg.15.6.409

5. Talbot K, Davies KE (2001) Spinal muscular atrophy. Semin Neurol 21(2):189-197. https://doi.org/10.1055/s-2001-15264

6. Monani UR, Lorson CL, Parsons DW, Prior TW, Androphy EJ, Burghes AH, McPherson JD (1999) A single nucleotide difference that alters splicing patterns distinguishes the SMA gene SMN1 from the copy gene SMN2. Hum Mol Genet 8(7):1177-1183. https://doi.org/10.1093/hmg/8.7.1177

7. Wirth B (2000) An update of the mutation spectrum of the survival motor neuron gene (SMN1) in autosomal recessive spinal muscular atrophy (SMA). Hum Mutat 15(3):228-237. https://doi.org/10.1002/ (SICI)1098-1004(200003)15:3<228::AID-HUMU3>3.0.CO;2-9

8. Ogino S, Leonard DG, Rennert H, Ewens WJ, Wilson RB (2002) Genetic risk assessment in carrier testing for spinal muscular atrophy. Am J Med Genet 110(4):301-307. https://doi.org/10.1002/ ajmg.10425

9. Frugier T, Nicole S, Cifuentes-Diaz C, Melki J (2002) The molecular bases of spinal muscular atrophy. Curr Opin Genet Dev 12(3): 294-298. https://doi.org/10.1016/S0959-437X(02)00301-5

10. Finkel RS (2013) Electrophysiological and motor function scale association in a pre-symptomatic infant with spinal muscular atrophy type I. Neuromuscul Disord 23(2):112-115. https://doi.org/10. 1016/j.nmd.2012.09.006

11. Sumner C, Paushkin S, Ko CP. Spinal muscular atrophy disease mechanisms and therapy. 1st Edition ISBN: 9780128036853 Academic Press 4th November 2016, Transcriptional and Splicing Regulation of Spinal Muscular Atrophy Genes N.N. Singh, M.D. Howell, R.N. Singh cap 5, 75-97

12. Kolb SJ, Coffey CS, Yankey JW, Krosschell K, Arnold WD, Rutkove SB, Swoboda KJ, Reyna SP, Sakonju A, Darras BT, Shell R, Kuntz N, Castro D, Parsons J, Connolly AM, Chiriboga CA, McDonald C, Burnette WB, Werner K, Thangarajh M, Shieh PB, Finanger E, Cudkowicz ME, McGovern MM, McNeil DE, Finkel R, Iannaccone ST, Kaye E, Kingsley A, Renusch SR, McGovern VL, Wang X, Zaworski PG, Prior TW, Burghes AHM, Bartlett A, Kissel JT, NeuroNEXT Clinical Trial Network on behalf of the NN101 SMA Biomarker Investigators (2017) Natural history of infantile-onset spinal muscular atrophy. Ann Neurol. https://doi.org/10.1002/ana.25101

13. Swoboda KJ, Prior TW, Scott CB, NcNaught TP, Wride MC, Reyna SP (2005) Natural history of denervation in SMA: relation to age, SMN2 copy number, and function. Ann Neurol 57(5):704-712. https://doi.org/10.1002/ana.20473

14. Soler-Botija C, Ferrer I, Gich I, Baiget M, Tizzano EF (2002) Neuronal death is enhanced and begins during foetal development in type I spinal muscular atrophy spinal cord. Brain 125(Pt. 7): 1624-1634. https://doi.org/10.1093/brain/awf155

15. Simic G, Seso-Simic D, Lucassen PJ, Islam A, Krsnik Z, Cviko A, Jelasic D, Barisic N et al (2000) Ultrastructural analysis and TUNEL demonstrate motor neuron apoptosis in WerdnigHoffmann disease. J Neuropathol Exp Neurol 59(5):398-407. https://doi.org/10.1093/jnen/59.5.398

16. Sumner C, Paushkin S, Ko CP. Spinal muscular atrophy disease mechanisms and therapy. 1st Edition ISBN: 9780128036853 Academic Press 4th November 2016, Developmental Aspects and Pathological Findings in Spinal Muscular Atrophy M.J. PérezGarcía1, L. Kong2, C.J. Sumner2, E.F. Tizzano1,3 cap 2, 21-42

17. Kaufmann P, McDermott MP, Darras BT, Finkel R, Kang P, Oskoui M, Constantinescu A, Sproule DM et al(2011) Observational study of spinal muscular atrophy type 2 and 3: functional outcomes over 1 year. Arch Neurol 68(6):779-786. https://doi.org/10.1001/ archneurol.2010.373

18. Hao le T, Duy PQ, Jontes JD, Wolman M, Granato M, Beattie CE (2013) Temporal requirement for SMN in motoneuron development. Hum Mol Genet 22(13):2612-2625. https://doi.org/10. 1093/hmg/ddt110

19. Murray LM, Lee S, Bäumer D, Parson SH, Talbot K, Gillingwater TH (2010) Pre-symptomatic development of lower motor neuron connectivity in a mouse model of severe spinal muscular atrophy. Hum Mol Genet 19(3):420-433. https://doi.org/10.1093/hmg/ ddp506

20. McGovern VL, Gavrilina TO, Beattie CE, Burghes AH (2008) Embryonic motor axon development in the severe SMA mouse. Hum Mol Genet 17(18):2900-2909. https://doi.org/10.1093/hmg/ ddn189

21. Le TT, McGovern VL, Alwine IE, Wang X, Massoni-Laporte A, Rich MM, Burghes AH (2011) Temporal requirement for high SMN expression in SMA mice. Hum Mol Genet 20(18):35783591. https://doi.org/10.1093/hmg/ddr275

22. Lutz CM, Kariya S, Patruni S, Osborne MA, Liu D, Henderson CE, Li DK, Pellizzoni L et al (2011) Postsymptomatic restoration of SMN rescues the disease phenotype in a mouse model of severe spinal muscular atrophy. J Clin Invest 121(8):3029-3041. https:// doi.org/10.1172/JCI57291

23. Gray SJ, Matagne V, Bachaboina L, Yadav S, Ojeda SR, Samulski RJ(2011) Preclinical differences of intravascular AAV9delivery to neurons and glia: a comparative study of adult mice and nonhuman primates. Mol Ther 19(6):1058-1069. https://doi.org/10.1038/mt. 2011.72

24. Kariya S, Park GH, Maeno-Hikichi Y, Leykekhman O, Lutz C, Arkovitz MS, Landmesser LT, Monani UR (2008) Reduced SMN proteinimpairs maturation of theneuromuscularjunctionsinmouse models of spinal muscular atrophy. Hum Mol Genet 17(16):25522569. https://doi.org/10.1093/hmg/ddn156

25. Bogdanik LP, Osborne MA, Davis C, Martin WP, Austin A, Rigo F, Bennett CF, Lutz CM (2015) Systemic, postsymptomatic antisense oligonucleotide rescues motor unit maturation delay in a new mouse model for type II/III spinal muscular atrophy. Proc Natl Acad Sci U S A 112(43):E5863-E5872. https://doi.org/10.1073/ pnas.1509758112

26. Zhou H, Meng J, Marrosu E, Janghra N, Morgan J, Muntoni F (2015) Repeated low doses of morpholino antisense oligomer: an intermediatemouse model of spinal muscularatrophy to explore the window of therapeutic response. Hum Mol Genet 24(22):62656277. https://doi.org/10.1093/hmg/ddv329

27. Feng Z, Ling KK, Zhao X, Zhou C, Karp G, Welch EM, Naryshkin N, Ratni Het al (2016) Pharmacologically induced mouse model of adult spinal muscular atrophy to evaluate effectiveness of therapeutics after disease onset. Hum Mol Genet 25(5):964-975. https://doi. org/10.1093/hmg/ddv629

28. Foust KD, Nurre E, Montgomery CL, Hernandez A, Chan CM, Kaspar BK (2009) Intravascular AAV9 preferentially targets neonatal neurons and adult astrocytes. Nat Biotechnol 27(1):59-65. https://doi.org/10.1038/nbt.1515

29. Foust KD, Wang X, McGovern VL, Braun L, Bevan AK, Haidet AM, Le TT, Morales PR et al (2010) Rescue of the spinal muscular atrophy phenotype in a mouse model by early postnatal delivery of SMN. Nat Biotechnol 28(3):271-274. https://doi.org/10.1038/nbt.1610

30. Passini MA, Bu J, Richards AM, Treleaven CM, Sullivan JA, O'Riordan CR, Scaria A, Kells AP et al (2014) Translational fidelity of intrathecal delivery of self-complementary AAV9-survival motor neuron 1 for spinal muscular atrophy. Hum Gene Ther 25(7):619-630. https://doi.org/10.1089/hum.2014.011

31. Bevan AK, Duque S, Foust KD, Morales PR, Braun L, Schmelzer L, Chan CM, McCrate M et al (2011) Systemic gene delivery in large species for targeting spinal cord, brain, and peripheral tissues 
for pediatric disorders. Mol Ther 19(11):1971-1980. https://doi. org/10.1038/mt.2011.157

32. Gray SJ, Nagabhushan Kalburgi S, McCown TJ, Samulski RJ (2013) Global CNS gene delivery and evasion of anti-AAV-neutralizing antibodies by intrathecal AAV administration in non-human primates. Gene Ther 20(4):450-459. Erratum in: Gene Ther 2013 Apr;20(4):465. https://doi.org/10.1038/gt.2012.101

33. Samaranch L, Salegio EA, San Sebastian W, Kells AP, Foust KD, Bringas JR, Lamarre C, Forsayeth J et al (2012) Adeno-associated virus serotype 9 transduction in the central nervous system of nonhuman primates. Hum Gene Ther 23(4):382-389. https://doi.org/ 10.1089/hum.2011.200

34. Samaranch L, Salegio EA, San Sebastian W, Kells AP, Bringas JR, Forsayeth J, Bankiewicz KS (2013) Strong cortical and spinal cord transduction after AAV7 and AAV9 delivery into the cerebrospinal fluid of nonhuman primates. Hum Gene Ther 24(5):526-532. https://doi.org/10.1089/hum.2013.005

35. Meyer K, Ferraiuolo L, Schmelzer L, Braun L, McGovern V, Likhite S, Michels O, Govoni A et al (2015) Improving single injection CSF delivery of AAV9-mediated gene therapy for SMA - a dose response study in mice and nonhuman primates. Mol Ther 23(3):477-487. https://doi.org/10.1038/mt.2014.210

36. Federici T, Taub JS, Baum GR, Gray SJ, Grieger JC, Matthews KA, Handy CR, Passini MA et al (2012) Robust spinal motor neuron transduction following intrathecal delivery of AAV9 in pigs. Gene Ther 19(8):852-859. https://doi.org/10.1038/gt.2011.130

37. Duque SI, Arnold WD, Odermatt P, Li X, Porensky PN, Schmelzer L, Meyer K, Kolb SJ et al (2015) A large animal model of spinal muscular atrophy and correction of phenotype. Ann Neurol 77(3): 399-414. https://doi.org/10.1002/ana.24332

38. Chiriboga CA, Swoboda KJ, Darras BT, Iannaccone ST, Montes J, De Vivo DC, Norris DA, Bennett CF et al (2016) Results from a phase 1 study of nusinersen(ISIS-SMN(Rx)) in children with spinal muscular atrophy. Neurology 86(10):890-897. https://doi.org/10. 1212/WNL.0000000000002445

39. Finkel RS, Chiriboga CA, Vajsar J, Day JW, Montes J, De Vivo DC, Yamashita M, Rigo F et al (2016) Treatment of infantile-onset spinal muscular atrophy with nusinersen: a phase 2, open-label, dose-escalation study. Lancet 388(10063):3017-3026. https://doi. org/10.1016/S0140-6736(16)31408-8

40. Finkel RS, Mercuri E, Darras BT, Connolly AM, Kuntz NL, Kirschner J, Chiriboga CA, Saito K et al (2017) Nusinersen versus sham control in infantile-onset spinal muscular atrophy. N Engl J Med 377(18):17 23-1173. https://do i.org/10.1056/ NEJMoa1702752
41. Mendell JR, Al-Zaidy S, Shell R, Arnold WD, Rodino-Klapac LR, Prior TW, Lowes L, Alfano L et al (2017) Single-dose gene-replacement therapy for spinal muscular atrophy. N Engl J Med 377(18):1713-1722. https://doi.org/10.1056/NEJMoa1706198

42. Sulaiman W, Gordon T (2013) Neurobiology of peripheral nerve injury, regeneration, and functional recovery: from bench top research to bedside application. Ochsner J 13(1):100-108

43. Domingues-Faria C, Vasson MP, Goncalves-Mendes N, Boirie Y, Walrand S (2016) Skeletal muscle regeneration and impact of aging and nutrition. Ageing Res Rev 26:22-36. https://doi.org/10.1016/j. arr.2015.12.004

44. DarabidH, Perez-Gonzalez AP, Robitaille R(2014)Neuromuscular synaptogenesis: coordinating partners with multiple functions. Nat Rev Neurosci 15(11):703-718. https://doi.org/10.1038/nrn3821

45. Bertini E, Dessaud E, Mercuri E, Muntoni F, Kirschner J, Reid C, Lusakowska A, Comi GP et al (2017) Safety and efficacy of olesoxime in patients with type 2 or non-ambulatory type 3 spinal muscular atrophy: a randomised, double-blind, placebo-controlled phase 2 trial. Lancet Neurol 16(7):513-522. https://doi.org/10. 1016/S1474-4422(17)30085-6

46. Niranjan V, Bach JR (1998) Noninvasive management of pediatric neuromuscular ventilatory failure. Crit Care Med 26(12):20612065

47. Bach JR, Saltstein K, Sinquee D, Weaver B, Komaroff E (2007) Long-term survival in Werdnig-Hoffmann disease. Am J Phys Med Rehabil 86(5):339-345 quiz 346-348, 379

48. Finkel RS, McDermott MP, Kaufmann P, Darras BT, Chung WK, Sproule DM, Kang PB, Foley AR et al (2014) Observational study of spinal muscular atrophy type I and implications for clinical trials. Neurology 83(9):810-817. https://doi.org/10.1212/WNL. 0000000000000741

49. Arnold WD, Burghes AH (2013) Spinal muscular atrophy: development and implementation of potential treatments. Ann Neurol 74(3):348-362. https://doi.org/10.1002/ana.23995

50. Glanzman AM, Mazzone E, Main M, Pelliccioni M, Wood J, Swoboda KJ, Scott C, Pane M et al (2010) The Children's Hospital of Philadelphia Infant Test of Neuromuscular Disorders (CHOP INTEND): test development and reliability. Neuromuscul Disord 20(3):155-161. https://doi.org/10.1016/j.nmd.2009.11.014

51. Nizzardo M, Simone C, Rizzo F, Salani S, Dametti S, Rinchetti P, Del Bo R, Foust K et al (2015) Gene therapy rescues disease phenotype in a spinal muscular atrophy with respiratory distress type 1 (SMARD1) mouse model. Science Ad 1(2):e1500078. https://doi. org/10.1126/sciadv.1500078 